\title{
A Riesz type representation for lower semi-continuous, monotone, local functionals on $C_{c}(X)^{+}$
}

\author{
Ralph Chill \\ R. Chill, Institut für Analysis, Fachrichtung Mathematik, TU Dresden, 01062 Dresden, Germany
}

Mahamadi Warma

M. Warma, University of Puerto Rico, Faculty of Natural Sciences, Department of Mathematics (Rio Piedras Campus), PO Box 70377 San Juan PR 00936-8377 (USA)

\begin{abstract}
We prove a Riesz type representation theorem for lower semi-continuous, monotone, local functionals on $C_{c}(X)^{+}$,
\end{abstract} where $X$ is a locally compact, separable, metric space.

Keywords: Lower semi-continuous functional, monotone functional, local functional, locally compact metric space, Riesz representation theorem.

2000 MSC: 28C15, 46E05, 28C99

\section{Introduction}

By the classical Riesz representation theorem, linear and continuous functionals on spaces of continuous functions are represented by regular Borel measures. More precisely, if $X$ is a locally compact Hausdorff space, then every linear, continuous functional $\varphi: C_{c}(X) \rightarrow \mathbb{R}$ is of the form

$$
\varphi(u)=\int_{X} u \mathrm{~d} \mu \quad\left(u \in C_{c}(X)\right)
$$

for some regular (locally bounded) Borel measure $\mu$ on $X$. Tikhonov \& Arsenin [9] were perhaps the first to state a similar representation theorem for in general nonlinear, continuous functionals $\varphi: C([0,1]) \rightarrow \mathbb{R}$ which are monotone and local; local means here that $\varphi(u+v)=\varphi(u)+\varphi(v)$ for all pairs $u, v$ of continuous functions with disjoint support (in their article and several subsequent articles such functionals are called additive or disjointly additive). Tikhonov $\&$ Arsenin proved that such functionals are of the form

$$
\varphi(u)=\int_{0}^{1} B(x, u(x)) \mathrm{d} \mu \quad(u \in C([0,1]))
$$

for some Borel measure $\mu$ and some function $B$ which is measurable in the first variable and continuous and monotone in the second variable. Independently, Chacon \& Friedman [3] and Friedman \& Katz [6, 7] generalized the Riesz representation theorem in a slightly different way. Instead of monotone and local functionals they considered strongly additive functionals, first on $C([0,1])$ and then on $C(X)$ where $X$ is a compact, metric space, or, respectively, a compact Hausdorff space; for the definition of strong additivity, see [3, 6, 7]. For an account of these representation theorems, for representation theorems of Banach space valued functionals and other representation theorems on spaces of measurable functions we refer to Rao [8] and the references therein.

In this short note we state and prove a Riesz type representation theorem for lower semi-continuous, monotone, local functionals $\varphi: C_{c}(X)^{+} \rightarrow[0,+\infty]$. Actually, our functionals satisfy an additional condition (see condition (3) below) which is between locality / additivity (as considered by Tikhonov and Arsenin) and strong additivity (as 
considered by Chacon, Friedman and Katz). We think that a variant of the Tikhonov-Arsenin result or the ChaconFriedman-Katz results for the class of lower semi-continuous functionals is interesting as such, but we point out that our main theorem is also motivated by the fact that lower semi-continuous functionals naturally arise in the theory of abstract gradient systems; see [2] for the general theory, and [4] for a particular application of the Riesz type representation theorem to nonlinear parabolic boundary value problems.

\section{The result}

Let $(X, d)$ be a locally compact, separable, metric space, let $C_{c}(X)$ be the space of continuous functions with compact support in $X$ (equipped with the usual topology, that is, the inductive limit topology, so that convergence of a sequence or net of elements in $C_{c}(X)$ means that all these elements have their support in one compact set and that they converge uniformly on $X$ ), and let $C_{c}(X)^{+}$be the positive cone in $C_{c}(X)$. Given a functional $\varphi: C_{c}(X)^{+} \rightarrow[0,+\infty]$, we call $D(\varphi)=\left\{u \in C_{c}(X)^{+}: \varphi(u)<+\infty\right\}$ its effective domain. The effective support of the functional $\varphi$ is the set

$$
\begin{aligned}
& \operatorname{supp}[\varphi]:=X \backslash\{x \in X: \text { there exists a neighbourhood } U \text { of } x \text { such that for every } \\
& \qquad u \in D(\varphi) \text { with } \operatorname{supp}[u] \subseteq U \text { one has } \varphi(u)=0\} .
\end{aligned}
$$

We say that the functional $\varphi$ is monotone if for every $u, v \in C_{c}(X)^{+}$

$$
u \leq v \Rightarrow \varphi(u) \leq \varphi(v)
$$

and we call it local if for every $u, v \in C_{c}(X)^{+}$

$$
\operatorname{supp}[u] \cap \operatorname{supp}[v]=\emptyset \quad \Rightarrow \quad \varphi(u+v)=\varphi(u)+\varphi(v) .
$$

Theorem 1. For every functional $\varphi: C_{c}(X)^{+} \rightarrow[0,+\infty]$ the following assertions (I) and (II) are equivalent.

(I) The functional $\varphi$ is lower semi-continuous, monotone, local and for every $u, v \in D(\varphi)$, one has $u \vee v, u \wedge v \in$ $D(\varphi)$ and

$$
\varphi(u \vee v)+\varphi(u \wedge v) \leq \varphi(u)+\varphi(v)
$$

where $u \vee v($ resp. $u \wedge v)$ denote the (pointwise) supremum (resp. infimum) of the functions $u$ and $v$.

(II) There exist a finite, regular Borel measure $\mu$ on $X$ with $\operatorname{supp}[\mu] \subseteq \operatorname{supp}[\varphi]$ and a function $B: X \times \mathbb{R} \rightarrow[0,+\infty]$ such that,

(i) $B(\cdot, s)$ is a measurable function for each $s$,

(ii) $B(x, 0)=0$ and $B(x, \cdot)$ is monotone and lower semi-continuous for $\mu$-a.e. $x \in X$,

(iii) $\varphi(u)=\int_{X} B(x, u(x)) \mathrm{d} \mu$ for every $u \in D(\varphi)$.

Remark 2. (a) We point out that the representing measure $\mu$ and function $B$ are not unique. For example, given a representing measure $\mu$ and a representing function $B$, and given any Borel measurable weight $w: X \rightarrow \mathbb{R}_{+}$ which is bounded from above and from below (away from zero), the weighted measure w $\mathrm{d} \mu$ and the function $B / w$ represent $\varphi$, too.

(b) On the other hand, the proof of the Theorem shows that for any pair $\varphi_{1}, \varphi_{2}$ of lower semi-continuous, monotone, local functionals satisfying the inequality (3) one can find a common representing measure $\mu$ with $\operatorname{supp}[\mu] \subseteq \operatorname{supp}\left[\varphi_{1}\right] \cup \operatorname{supp}\left[\varphi_{2}\right]$ and two representing functions $B_{1}$ and $B_{2}$ satisfying the conditions (II) (i)-(iii) of the Theorem. It suffices to take for example $\mu=\mu_{1}+\mu_{2}$, if $\mu_{1}$ and $\mu_{2}$ are the representing measures the existence of which is guaranteed by the Theorem. 


\section{Proof of the Theorem}

We start by proving the implication in the Theorem which is relatively straightforward.

Proof (Proof Of The Theorem, (II) $\Rightarrow(\mathrm{I})$ ). Assume that Assertion (II) holds. Note that for every $u \in C_{c}(X)^{+}$ the function $B(\cdot, u(\cdot))$ is measurable due to assumptions (II)(i) and (II)(ii). In particular, the integral in (II)(iii) is welldefined. The monotonicity of the function $B(x, \cdot)$ (for $\mu$-almost every $x \in X$, assumption (II)(i)) and the monotonicity of the integral imply that the functional $\varphi$ is monotone. We show that it is lower semi-continuous. Let $\left(u_{n}\right) \subseteq C_{c}(X)^{+}$ be a sequence which converges to $u \in C_{c}(X)^{+}$. Then $u_{n}$ converges to $u$ everywhere. Since, for $\mu$-almost every $x \in X$, the function $B(x, \cdot)$ is lower semi-continuous, we obtain $B(x, u(x)) \leq \liminf _{n \rightarrow \infty} B\left(x, u_{n}(x)\right)$ for $\mu$-almost every $x \in X$. Using Fatou's lemma, we therefore obtain that

$$
\varphi(u)=\int_{X} B(x, u) \mathrm{d} \mu \leq \int_{X} \liminf _{n \rightarrow \infty} B\left(x, u_{n}(x)\right) \mathrm{d} \mu \leq \liminf _{n \rightarrow \infty} \int_{X} B\left(x, u_{n}(x)\right) \mathrm{d} \mu=\liminf _{n \rightarrow \infty} \varphi\left(u_{n}\right) .
$$

Hence, $\varphi$ is lower semi-continuous.

Let $u, v \in D(\varphi) \subseteq C_{c}(X)^{+}$. Clearly, $u \vee v, u \wedge v \in C_{c}(X)^{+}$. From the equality

$$
\begin{aligned}
\varphi(u \vee v)+\varphi(u \wedge v)= & \int_{X} B(x, u \vee v) \mathrm{d} \mu+\int_{X} B(x, u \wedge v) \mathrm{d} \mu \\
= & \int_{\{u \leq v\}} B(x, v) \mathrm{d} \mu+\int_{\{u \leq v\}} B(x, u) \mathrm{d} \mu \\
& +\int_{\{u>v\}} B(x, u) \mathrm{d} \mu+\int_{\{u>v\}} B(x, v) \mathrm{d} \mu \\
= & \int_{X} B(x, u) \mathrm{d} \mu+\int_{X} B(x, v) \mathrm{d} \mu \\
= & \varphi(u)+\varphi(v)
\end{aligned}
$$

we obtain that $u \vee v, u \wedge v \in D(\varphi)$ and (3) holds (even with equality). The fact that $\varphi$ is local follows immediately from condition (3) by noticing that $\varphi(u+v)=\varphi(u \vee v)$ and $0=\varphi(0)=\varphi(u \wedge v)$ for every $u, v \in C_{c}(X)^{+}$with disjoint support.

To prove the converse implication (I) $\Rightarrow$ (II), we proceed stepwise, in the form of several lemmas.

Throughout the following, we denote the Borel sets of $X$ by $\mathscr{B}$ and the class of compact subsets of $X$ is denoted by $\mathscr{K}$. We assume also that the functional $\varphi$ satisfies the condition (I) in the Theorem.

For $\delta>0$ and every subset $K \subseteq X$ we define

$$
K^{\delta}:=\{x \in X: d(x, K) \leq \delta\} .
$$

With this definition, for every compact subset $K \in \mathscr{K}$ we define

$$
\mathscr{R}(K):=\left\{\rho \in C_{c}(X)^{+}: \text {there exists } \delta>0 \text { such that } \rho \geq 1 \text { on } K^{\delta}\right\} .
$$

Definition 3. For each $u \in D(\varphi)$ we define a nonnegative set function $\mu_{u}(\cdot)$ on $\mathscr{K}$ by setting

$$
\mu_{u}(K)=\inf _{\rho \in \mathscr{R}(K)} \varphi(u \rho) \quad(K \in \mathscr{K}) .
$$

We remark that for every $\rho \in \mathscr{R}(K)$ one has $\rho \wedge 1 \in \mathscr{R}(K)$. Therefore, in the definition of $\mu_{u}(K)$ it suffices to take the infimum over all functions $\rho \in \mathscr{R}(K)$ which are $=1$ on some $K^{\delta}$. In particular, by the monotonicity of $\varphi$, $\mu_{u}(K) \leq \varphi(u)<+\infty$ for every compact $K \subseteq X$.

Lemma 4 (Finite additivity). Let $u \in D(\varphi)$, and let $K_{1}, K_{2} \subseteq X$ be two compact sets such that $K_{1} \cap K_{2}=\emptyset$. Then

$$
\mu_{u}\left(K_{1} \cup K_{2}\right)=\mu_{u}\left(K_{1}\right)+\mu_{u}\left(K_{2}\right) .
$$


Proof. Let $K_{1}, K_{2} \subseteq X$ be two compact sets such that $K_{1} \cap K_{2}=\emptyset$. Then $d\left(K_{1}, K_{2}\right)=: r>0$. We can find two functions $\rho_{i} \in \mathscr{R}\left(K_{i}\right)(i=1,2)$ such that $0 \leq \rho_{i} \leq 1$ and $\operatorname{supp}\left[\rho_{1}\right] \cap \operatorname{supp}\left[\rho_{2}\right]=\emptyset$; think of functions $\rho_{i}=\rho_{i}(x)$ which depend in fact only on the distance dist $\left(x, K_{i}\right)$.

Let $\rho \in \mathscr{R}\left(K_{1} \cup K_{2}\right)$. Since $\operatorname{supp}\left[u \rho \rho_{i}\right] \subseteq \operatorname{supp}\left[\rho_{i}\right]$, it follows that $\operatorname{supp}\left[u \rho \rho_{1}\right] \cap \operatorname{supp}\left[u \rho \rho_{2}\right]=\emptyset$. The monotonicity and locality of $\varphi$ implies

$$
\begin{aligned}
\varphi(u \rho) & \geq \varphi\left(u \rho\left(\rho_{1}+\rho_{2}\right)\right) \\
& =\varphi\left(u \rho \rho_{1}\right)+\varphi\left(u \rho \rho_{2}\right) \\
& \geq \mu_{u}\left(K_{1}\right)+\mu_{u}\left(K_{2}\right) .
\end{aligned}
$$

Since $\rho \in \mathscr{R}\left(K_{1} \cup K_{2}\right)$ was arbitrary, this implies $\mu_{u}\left(K_{1} \cup K_{2}\right) \geq \mu_{u}\left(K_{1}\right)+\mu_{u}\left(K_{2}\right)$.

Now let $\rho_{i}^{\prime} \in \mathscr{R}\left(K_{i}\right)(i=1,2)$. Then, again by monotonicity and locality,

$$
\begin{aligned}
\varphi\left(u \rho_{1}^{\prime}\right)+\varphi\left(u \rho_{2}^{\prime}\right) & \geq \varphi\left(u \rho_{1}^{\prime} \rho_{1}\right)+\varphi\left(u \rho_{2}^{\prime} \rho_{2}\right) \\
& =\varphi\left(u\left(\rho_{1}^{\prime} \rho_{1}+\rho_{2}^{\prime} \rho_{2}\right)\right) \\
& \geq \mu_{u}\left(K_{1} \cup K_{2}\right) .
\end{aligned}
$$

Since $\rho_{i}^{\prime} \in \mathscr{R}\left(K_{i}\right)(i=1,2)$ were arbitrary, this implies $\mu_{u}\left(K_{1}\right)+\mu_{u}\left(K_{2}\right) \geq \mu_{u}\left(K_{1} \cup K_{2}\right)$.

Lemma 5 (Monotonicity). Let $u \in D(\varphi)$ and let $K_{1}, K_{2} \subseteq X$ be two compact sets such that $K_{1} \subseteq K_{2}$. Then

$$
\mu_{u}\left(K_{1}\right) \leq \mu_{u}\left(K_{2}\right) .
$$

PROOF. This follows immediately from the definition of $\mu_{u}$ and the monotonicity of $\varphi$.

Lemma 6. Let $u \in D(\varphi)$ and let $K_{1}, K_{2} \subseteq X$ be two compact sets. Then

$$
\mu_{u}\left(K_{1} \cup K_{2}\right)+\mu_{u}\left(K_{1} \cap K_{2}\right) \leq \mu_{u}\left(K_{1}\right)+\mu_{u}\left(K_{2}\right) .
$$

PRoof. Let $\rho_{i} \in \mathscr{R}\left(K_{i}\right)(i=1,2)$. Then, by assumption (3),

$$
\begin{aligned}
\varphi\left(u \rho_{1}\right)+\varphi\left(u \rho_{2}\right) & \geq \varphi\left(u\left(\rho_{1} \vee \rho_{2}\right)\right)+\varphi\left(u\left(\rho_{1} \wedge \rho_{2}\right)\right. \\
& \geq \mu_{u}\left(K_{1} \cup K_{2}\right)+\mu_{u}\left(K_{1} \cap K_{2}\right) .
\end{aligned}
$$

Since $\rho_{i} \in \mathscr{R}\left(K_{i}\right)(i=1,2)$ were arbitrary, this implies $\mu_{u}\left(K_{1}\right)+\mu_{u}\left(K_{2}\right) \geq \mu_{u}\left(K_{1} \cup K_{2}\right)+\mu_{u}\left(K_{1} \cap K_{2}\right)$.

Lemma 7 (Outer regularity). Let $u \in D(\varphi)$, let $\left(K_{m}\right)$ be a decreasing sequence of compact subsets of $X$, and let $K:=\bigcap_{m} K_{m}$. Then

$$
\lim _{m \rightarrow \infty} \mu_{u}\left(K_{m}\right)=\mu_{u}(K) .
$$

Proof. First, the monotonicity of $\mu_{u}$ (Lemma 5) implies that $\lim _{m \rightarrow \infty} \mu_{u}\left(K_{m}\right) \geq \mu_{u}(K)$. In order to prove the converse inequality, observe that for every $\delta, \delta^{\prime}>0$ with $\delta>\delta^{\prime}$ there exists $m_{0} \in \mathbb{N}$ such that for every $m \geq m_{0}$ one has $K_{m}^{\delta^{\prime}} \subseteq K^{\delta}$ (here we use that $\left(K_{m}\right)$ is decreasing and $K=\bigcap_{m} K_{m}$ ). In particular, for every $\rho \in \mathscr{R}(K)$ there exists $m_{0} \in \mathbb{N}$ such that for every $m \geq m_{0}$ one has $\rho \in \mathscr{R}\left(K_{m}\right)$. As a consequence

$$
\varphi(u \rho) \geq \mu_{u}\left(K_{m}\right) \text { for every } m \geq m_{0},
$$

or,

$$
\varphi(u \rho) \geq \lim _{m \rightarrow \infty} \mu_{u}\left(K_{m}\right) .
$$

Since this inequality holds for every $\rho \in \mathscr{R}(K)$, this proves $\mu_{u}(K) \geq \lim _{m \rightarrow \infty} \mu_{u}\left(K_{m}\right)$.

Lemma 8. For every $u \in D(\varphi)$ the set function $\mu_{u}$ can be uniquely extended to a finite, regular Borel measure on $X$ (denoted again by $\mu_{u}$ in the following). Moreover, $\operatorname{supp}\left[\mu_{u}\right] \subseteq \operatorname{supp}[\varphi]$. 
Proof. By Lemmas 4, 5, 6 and 7, $\mu_{u}$ is a regular content on $\mathscr{K}$. The fact that $\mu_{u}$ extends to a regular Borel measure (which we denote again by $\mu_{u}$ ) follows from standard measure theory, including the theory of measures on topological spaces (see, for example, [1, Kapitel I $\S 3$, Kapitel IV], [5, §416, and in particular Corollary 416M]). Note that $\mu_{u}(X)=\varphi(u)<+\infty$ for $u \in D(\varphi)$. The inclusion $\operatorname{supp}\left[\mu_{u}\right] \subseteq \operatorname{supp}[\varphi]$ is a straightforward consequence of the definition of $\mu_{u}$ and the definition of the effective support of $\varphi$.

Lemma 9 (Monotonicity of $\mu$.). Let $u, v \in D(\varphi)$ be such that $u \leq v$. Then $\mu_{u} \leq \mu_{v}$.

Proof. The monotonicity of $\varphi$ and the definition of the measures $\mu_{u}$ and $\mu_{v}$ imply $\mu_{u}(K) \leq \mu_{v}(K)$ for every compact subset $K \subseteq X$. The claim then follows from the inner regularity of $\mu_{u}$ and $\mu_{v}$.

Lemma 10. Let $\left(u_{n}\right) \subseteq D(\varphi)$ and $u \in D(\varphi)$ be such that $u_{n} \leq u$ and $\lim _{n \rightarrow \infty} u_{n}=u$ in $C_{c}(X)$. Then

$$
\lim _{n \rightarrow \infty} \mu_{u_{n}}(G)=\mu_{u}(G) \quad \text { for every } G \in \mathscr{B}
$$

Proof. By Lemma 9, the domination $u_{n} \leq u$ implies $\mu_{u}(G)-\mu_{u_{n}}(G) \geq 0$ for every $G \in \mathscr{B}$. Hence, for every $G \in \mathscr{B}$,

$$
\begin{aligned}
0 & \leq \limsup _{n \rightarrow \infty}\left(\mu_{u}(G)-\mu_{u_{n}}(G)\right) \\
& \leq \limsup _{n \rightarrow \infty}\left(\mu_{u}(G)-\mu_{u_{n}}(G)+\mu_{u}\left(G^{c}\right)-\mu_{u_{n}}\left(G^{c}\right)\right) \\
& =\limsup _{n \rightarrow \infty}\left(\mu_{u}(X)-\mu_{u_{n}}(X)\right) \\
& =\limsup _{n \rightarrow \infty}\left(\varphi(u)-\varphi\left(u_{n}\right)\right) \\
& \leq 0
\end{aligned}
$$

where in the last inequality we have used the lower semi-continuity of $\varphi$. The preceding chain of inequalities implies the claim.

Lemma 11. For every $u \in D(\varphi)$ one has $\mu_{u}(\{u=0\})=0$.

PROOF. First, for every compact set $K \subseteq X \backslash \operatorname{supp}[u]$ one has $\mu_{u}(K)=0$. This follows from the definition of $\mu_{u}$, from the fact that $X \backslash \operatorname{supp}[u]$ is open, and from $\varphi(0)=0$.

Now, we can find an increasing sequence $\left(u_{n}\right) \subseteq D(\varphi)$ such that $\lim _{n \rightarrow \infty} u_{n}=u$ in $C_{c}(X)$ and $u_{n}=0$ in a neighborhood of $\{u=0\}$. In particular, $\{u=0\} \subseteq X \backslash \operatorname{supp}\left[u_{n}\right]$. By the preceding argument, and by interior regularity, we have $\mu_{u_{n}}(\{u=0\})=0$ for every $n$. By Lemma 10, this implies $\mu_{u}(\{u=0\})=0$.

Lemma 12. Let $u, v \in D(\varphi)$. Then $\mu_{u}(G) \leq \mu_{v}(G)$ for every Borel set $G \subseteq\{u \leq v\}$.

Proof. Step 1: We first prove the inequality $\mu_{u}(G) \leq \mu_{v}(G)$ for every Borel set $G \subseteq\{u<v\}$. By continuity of $u$ and $v$, the set $\{u<v\}$ is open. Hence, for every compact set $K \subseteq\{u<v\}$ there exists $\delta>0$ such that $K^{\delta} \subseteq\{u<v\}$. It is then easy to see that the monotonicity of $\varphi$ and the definition of the measures $\mu_{u}$ and $\mu_{v}$ imply $\mu_{u}(K) \leq \mu_{v}(K)$ for every compact subset $K \subseteq G$. The inequality $\mu_{u}(G) \leq \mu_{v}(G)$ for Borel sets $G \subseteq\{u<v\}$ then follows from the inner regularity of $\mu_{u}$ and $\mu_{v}$.

Step 2: Let now $G$ be a Borel set in $\{u \leq v$ and $0<v\}$. Let $\left(\lambda_{n}\right) \subseteq \mathbb{R}_{+}$be a sequence such that $\lambda_{n}<1$ and $\lim _{n \rightarrow \infty} \lambda_{n}=1$. Then, for every $n$ one has

$$
\{u \leq v \text { and } 0<v\} \subseteq\left\{\lambda_{n} u<v\right\} .
$$

Hence, by Step 1 , for every $n$,

$$
\mu_{\lambda_{n} u}(G) \leq \mu_{v}(G)
$$

Clearly, $\lambda_{n} u \leq u$ and $\lim _{n \rightarrow \infty} \lambda_{n} u=u$ in $C_{c}(X)$. Hence, by Lemma 10,

$$
\mu_{u}(G)=\lim _{n \rightarrow \infty} \mu_{\lambda_{n} u}(G) \leq \mu_{v}(G) .
$$


Step 3: Finally, let $G$ be an arbitrary Borel set in $\{u \leq v\}$. Then, by Step 2 and by Lemma 11,

$$
\begin{aligned}
\mu_{u}(G) & =\mu_{u}(G \cap\{0<v\}) \\
& \leq \mu_{v}(G \cap\{0<v\}) \\
& =\mu_{v}(G),
\end{aligned}
$$

which is the claim.

In the remainder of this note, fix $\left(w_{n}\right) \subseteq D(\varphi)$ a dense subset of $D(\varphi)$ (this is possible since the space $C_{c}(X)$ is separable by assumption and [10]). Then we define a measure $\mu$ on $\mathscr{B}$ by

$$
\mu(G)=\sum_{n=1}^{\infty} \frac{1}{2^{n}} \frac{\mu_{w_{n}}(G)}{1+\mu_{w_{n}}(X)} \quad(G \in \mathscr{B}) .
$$

It is clear that $\mu$ is a finite, regular Borel measure on $X$.

Lemma 13. Every measure $\mu_{u}(u \in D(\varphi))$ is absolutely continuous with respect to $\mu$.

Proof. Let $G \in \mathscr{B}$ be such that $\mu(G)=0$. It follows from the definition of $\mu$ and the positivity of $\mu_{w_{n}}$ that $\mu_{w_{n}}(G)=0$ for every $n \in \mathbb{N}$.

Now let $u \in D(\varphi)$. There exists a subsequence $\left(w_{n_{k}}\right)$ such that $\lim _{k \rightarrow \infty} w_{n_{k}}=u$ in $C_{c}(X)$. Define $u_{k}:=w_{n_{k}} \wedge u$. Then $u_{k} \leq w_{n_{k}}$ and Lemma 9 implies that $\mu_{u_{k}}(G)=0$. Moreover, $u_{k} \leq u$ and $\lim _{k \rightarrow \infty} u_{k}=u$ in $C_{c}(X)$. From this and Lemma 10 we obtain $\mu_{u}(G)=0$. Hence, $\mu_{u}$ is absolutely continuous with respect to $\mu$.

By the preceding lemma and by the Radon-Nikodym theorem, for every $u \in D(\varphi)$ there exists a Borel measurable function $B_{u}=B_{u}(x)$ such that

$$
\mu_{u}(G)=\int_{G} B_{u}(x) \mathrm{d} \mu(x) \quad(G \in \mathscr{B})
$$

Lemma 14. Let $u_{n}, u, v \in D(\varphi)$. Then:

(a) If $u_{n} \leq u$ and $\lim _{n \rightarrow \infty} u_{n}=u$ in $C_{c}(X)$, then $\lim _{n \rightarrow \infty} B_{u_{n}}=B_{u} \mu$-almost everywhere.

(b) $B_{u}=0 \mu$-almost everywhere on $\{u=0\}$.

(c) $B_{u} \leq B_{v} \mu$-almost everywhere on $\{u \leq v\}$.

ProOF. This lemma is an immediate consequence of Lemma 10, Lemma 11 and Lemma 12.

For every $x \in X$ we define the sets $W(x):=\left\{w_{n}(x): n \in \mathbb{N}\right\}$ and $I(x):=\{u(x): u \in D(\varphi)\}$. Clearly, $W(x) \subseteq I(x)$. Then, for every $x \in X$ and every $s \in \mathbb{R}_{+}$we define

$$
B(x, s)= \begin{cases}\sup _{n} B_{w_{n}}(x) 1_{\left\{w_{n}<s\right\}}(x) & \text { if } s \in I(x), \\ +\infty & \text { if } s \notin I(x) .\end{cases}
$$

Lemma 15. The function $B: X \times \mathbb{R}_{+} \rightarrow[0,+\infty]$ defined above satisfies the hypotheses II ( $i$ ) and II (ii) of the Theorem. Moreover,

(iv) for every $u \in D(\varphi)$ one has $B(\cdot, u(\cdot))=B_{u}(\cdot) \mu$-almost everywhere on $X$.

PROOF. First, for every $s \in \mathbb{R}_{+}$the set $\{x \in X: s \in I(x)\}=\left\{x \in X: s \leq \sup _{n} w_{n}(x)\right\}$ is a Borel set. From this and from the definition of $B$ one obtains that for every $s \in \mathbb{R}_{+}$the function $B(\cdot, s)$ is measurable. Thus, $B$ satisfies hypothesis II (i) of the Theorem.

Second, it follows readily from the definition of $B$ that $B(x, 0)=0$ for every $x \in X$ (since the sets $\left\{w_{n}<0\right\}$ are empty and therefore $1_{\left\{w_{n}<0\right\}}=0$ for every $n$ ). Moreover, since the sets $\left\{w_{n}<s\right\}$ are increasing with $s \in \mathbb{R}_{+}$, 
the function $B(x, \cdot)$ is monotone for every $x \in X$. Finally, for every $x \in X$, every $s \in I(x) \backslash\{0\}$ and every $\varepsilon>0$ there exists, by definition of the supremum, $n$ such that $w_{n}(x)<s$ and $B(x, s)-\varepsilon \leq B_{w_{n}}(x) \leq B(x, s)$. This implies $B(x, s)-\varepsilon \leq B\left(x, s^{\prime}\right) \leq B(x, s)$ for every $w_{n}(x)<s^{\prime} \leq s$. As a consequence, $B(x, \cdot)$ is lower semi-continuous for every $x \in X$. Thus, $B$ satisfies hypothesis II (ii) of the Theorem.

Third, we show the property (iv). Let $u \in D(\varphi)$. By Lemma 14 (c), there exists a set $A_{u}$ of $\mu$-measure zero such that for every $n$ and every $x \in\left\{w_{n} \leq u\right\} \backslash A_{u}$ one has $B_{w_{n}}(x) \leq B_{u}(x)$. As a consequence, $B(\cdot, u(\cdot)) \leq B_{u}(\cdot) \mu$-almost everywhere on $X$. In order to show the converse inequality we first note that, by Lemma 14 (b), $B_{u}(\cdot)=0=B(x, 0)$ $\mu$-almost everywhere on $\{u=0\}$. Hence, it remains to show that the inequality $B(\cdot, u(\cdot)) \geq B_{u}(\cdot)$ holds almost everywhere on $\{u>0\}$.

Let $\left(\lambda_{m}\right) \subseteq \mathbb{R}_{+}$be a sequence such that $\lambda_{m}<1$ and $\lim _{m \rightarrow \infty} \lambda_{m}=1$. Then, by Lemma 14 (c), there exists a set $A_{u}^{\prime}$ of $\mu$-measure zero such that for every $n, m$ and every $x \in\left\{\lambda_{m} u \leq w_{n}\right\} \backslash A_{u}^{\prime}$ one has $B_{\lambda_{m} u}(x) \leq B_{w_{n}}(x)$. Since $\lambda_{m} u<u$ on $\{u>0\}$ and since $W(x)$ is dense in $I(x)$ (for every $x \in X)$, this implies $B_{\lambda_{m} u}(x) \leq B(x, u(x))$ for every $m$ and every $x \in\{u>0\}$. Since $B_{\lambda_{m} u} \rightarrow B_{u} \mu$-almost everywhere on $X$ by Lemma 14 (a), we thus obtain the remaining inequality $B_{u}(\cdot) \leq B(\cdot, u(\cdot))$ on $\{u>0\}$.

Proof (Proof of the Theorem, (I) $\Rightarrow(\mathrm{II})$ ). Let $\left(w_{n}\right) \subseteq D(\varphi)$ be dense in $D(\varphi)$ and let $\mu$ be the Borel measure and $B: X \times \mathbb{R}_{+} \rightarrow[0,+\infty]$ be the function defined above. It follows from Lemma 8, that $\operatorname{supp}[\mu] \subseteq \operatorname{supp}[\varphi]$. By Lemma 15, the function $B$ satisfies the hypotheses II (i) and (ii) of the Theorem, and by property (iv) in Lemma 15 , for every $u \in D(\varphi)$ we have $B(\cdot, u(\cdot))=B_{u}(\cdot) \mu$-almost everywhere on $X$. By the definition of $B_{u}$ this means $\varphi(u)=\int_{X} B_{u}(x) \mathrm{d} \mu(x)=\int_{X} B(x, u(x)) \mathrm{d} \mu(x)$ for every $u \in D(\varphi)$. This just shows that $B$ satisfies hypothesis II (iii) of the Theorem. The Theorem is completely proved.

\section{References}

[1] H. Bauer, Maß- und Integrationstheorie, Walter de Gruyter, Berlin, New York, 1990

[2] H. Brezis, Opérateurs maximaux monotones. North-Holland, Amsterdam (1973).

[3] R. V. Chacon and N. Friedman. Additive functionals. Arch. Ration. Mech. Anal. 18 (1965), 230-240.

[4] R. Chill and M. Warma. Dirichlet and Neumann boundary conditions for the p-Laplace operator: What is in between? Proc. Royal Soc. Edinburgh Sect. A 142 (2012), 975-1002.

[5] D. H. Fremlin, Measure theory. Vol. 4, Topological measure spaces. Part I, Torres Fremlin, Colchester, 2006, Corrected second printing of the 2003 original.

[6] N. Friedman and M. Katz. A representation theorem for additive functionals. Arch. Ration. Mech. Anal. 21 (1966), $49-57$.

[7] N. Friedman and M. Katz. On additive functionals. Proc. Amer. Math. Soc. 21 (1969), 557-561.

[8] M. M. Rao Local functionals. Measure theory, Oberwolfach 1979 (Proc. Conf., Oberwolfach, 1979), pp. 484-496, Lecture Notes in Math., 794, Springer, Berlin, 1980.

[9] A. N. Tihonov and V. Ja. Arsenin, On some non-linear functionals, Mat. Sb. (N.S.) 65 (107) (1964), $512-521$.

[10] S. Warner, The topology of compact convergence on continuous function spaces, Duke Math. J. 25 (1958), $265-282$. 